\title{
Diagnóstico del consumo de energía eléctrica en la planificación de un sistema de gestión y norma técnica de calidad ISO 50001:2011
}

\author{
Yelenis M. Ladeuth, Danny D. López y Carlos A. Socarrás \\ Facultad de Ingeniería, Universidad de La Guajira, Km 5 salida Maicao, Riohacha-Colombia. \\ (correo-e: ymladeutho@uniguajira.edu.co; dlopezj@uniguajira.edu.co; csocarras@uniguajira.edu.co)
}

Recibido Jul. 17, 2020; Aceptado Sep. 14, 2020; Versión final Oct. 29, 2020, Publicado Feb. 2021

\begin{abstract}
Resumen
En este trabajo se presenta un diagnóstico para crear conciencia en el consumo de energía eléctrica al interior de la Universidad de La Guajira, sede Riohacha (Colombia). El diagnostico se realiza utilizando las facturas de energía del año 2015, 2016 y primer periodo del 2017. También, se utilizan un inventario de equipos electrónicos que existen en la universidad y los procesos tantos estratégicos, misionales de apoyo y evaluación. El desarrollo de la investigación fue de tipo descriptivo con diseño no experimental de acuerdo con lo propuesto en la NTC ISO 50001:2011, enmarcado en un sistema de mejora continua mediante el ciclo planear, hacer, verificar, actuar. Se determinó que el consumo de energía eléctrica en la Universidad de La Guajira es elevado por causa del uso irresponsable de equipos y maquinarias. Se concluye que se hace necesario implementar programas y modelos de seguimiento y monitoreo.
\end{abstract}

Palabras clave: consumo de energía; desempeño; planificación; ahorro; mejoramiento continuo

\section{Electrical energy consumption diagnosis for planning a quality and technical standard ISO 50001: 2011}

\begin{abstract}
This research study presents a diagnostic assessment to create awareness about electrical energy consumption at the University of La Guajira, Riohacha campus (Colombia). The diagnostic assessment is performed using university energy bills for the years 2015, 2016 and the first period of 2017. The assessment also examines an inventory of the university's electronic equipment, strategic processes, key support processes, and evaluation processes. This research study was descriptive and used a non-experimental design in accordance with what is proposed on the technical standard ISO 50001 : 2011. This standard is framed in a system of continuous improvement through a cycle (plan, do, verify, and act). It was determined that the consumption of electrical energy at the University of La Guajira was high due to irresponsible use of equipment and machinery. It is concluded that there is a need to implement monitoring programs and models.
\end{abstract}




\section{INTRODUCCIÓN}

El consumo de energía, según la unidad de planeación minero energética (UPME), ha presentado un incremento significativo considerando el tamaño de la población, el acelerado crecimiento industrial y otros factores que inciden de forma directa en este fenómeno, en donde se registra que para el año 2018, se obtuvo un consumo de energía de 69.121 GWh, con una diferencia respecto al año anterior de $6.019 \mathrm{GWh}$, representando un crecimiento de 3.3\%. Actualmente el gobierno nacional manifiesta su gran preocupación e invierte esfuerzos en presentar alternativas de mejora con base a la adopción de normas internacionales, que permitan de algún modo garantizar el acceso a bajos consumos de energía eléctrica. En concordancia con lo anteriormente expuesto, en la constitución política de 1991, en su artículo 80 estableció que el Estado planificaría el manejo y aprovechamiento de los recursos naturales, para garantizar su desarrollo sostenible, conservación, restauración o sustitución, y además este mismo se encargará de la prevención y el control de los factores de deterioro y la imposición de sanciones y la exigencia de la reparación de los daños causados al ambiente. Para (Robles, et al .2018) en su estudio, considera que los procedimientos de planificación energética, se realizan mediante una selección de un conjunto de criterios para tener en cuenta en proyectos de gestión para las zonas rurales.

La organización de las naciones unidas para el desarrollo Industrial( ONUDI), reconoció la importancia en dar respuesta al cambio climático y analizó las normas internacionales en el tema de energía, en el 2007 la ONUDI, solicito a la ISO desarrollar un estándar internacional de la gestión de la energía; en el 2008 se crea el comité de proyectos de gestión de energía y este se transformó en comité técnico en el 2011, donde posteriormente en junio de 2011 se hizo la publicación de la norma donde se estimó que podría influir en un $60 \%$ en el uso de la energía en el mundo. Es importante mencionar que el presente estudio toma de referencia la NTC ISO 50001 con versión 2011, porque en el momento que se desarrolló la investigación no se contaba con la actual versión que fue publicada en el 2018.

Se han realizado estudios enfocados a temas de eficiencia energética, donde diferentes autores exponen sus resultados, (Cabrera, et al. 2020) elaboró una herramienta para el análisis de sistema energético que establece estrategias y alternativas de planificación energética sistematizada. Mientras que (Wang, et al. 2019), estableció una metodología integrada basada en el análisis de fortalezas, debilidades, oportunidades y amenazas, que determina los factores y subfactores para la planificación energética sostenible. (Deveci, 2020) afirma que el proceso de planificación de la energía es crucial en temas de seguridad energética; han desarrollado investigaciones que involucran dos categorías, estudios de expansión de generación y tomas de decisiones con criterios múltiples (MCDM). (Solangi, 2019) se interesó en proponer una metodología integrada basada en el análisis de fortalezas, debilidades, oportunidades y amenazas, proceso de jerarquía analítica y técnica difusa que permite evaluar estrategias energéticas sostenibles. (García, et al. 2019) estudió acerca del consumo de energía eléctrica basado en la fabricación de cloques $\mathrm{H}-10$ donde se usó la herramienta estadística Statgraphics Centurion XV, para el monitoreo de la eficiencia energética asociada con el aumento de la producción.

(Bassani y Osorio 2017) analizaron la protección ambiental a través de la estrategia (yo soy así), ISO 50001, relacionada con la eficiencia de los sistemas de gestión de energía, como búsqueda de la optimización de los costos de producción de los bienes y la reducción de costos operativos que se encuentran en la cadena de valor organizacional. (Poveda, et al. 2019), presenta el contexto de mayor importancia de la NTC ISO 50001 donde se establecen los requisitos de gestión energética, el compromiso organizacional de implementar un modelo integral con enfoque de riesgo, para la contribución al éxito sostenible de las organizaciones para reducir la vulnerabilidad y riesgos asociados y (Krog, 2019), sugiere que se realicen investigaciones enfocadas a las estrategias y herramientas de implementación existentes con el propósito de identificar las que faltan en la planificación estratégica de la energía, para generar confiabilidad en los planes estratégicos de energía con éxitos.

"(Arévalo, 2016) afirma" que el diagnóstico inicial sobre el consumo es de gran importancia para iniciar el trabajo debido a que por medio de ellos se puede saber que potencialidades de ahorro se pueden tener en una empresa. Es importante resaltar que el consumo de energía en los sectores comercial e industrial, representa casi el $40 \%$ de las emisiones mundiales de gases de efectos invernadero. En este sentido, reducir este consumo es fundamental para que se cumplan los compromisos nacionales e internacionales; el estándar NTC ISO 50001 presenta un marco de referencia para la mejora continua con el propósito que las organizaciones reduzcan su consumo, además existen políticas alineadas a esta norma, pero lamentablemente no existe un proceso transparente que permita analizar los impactos de su implementación, como lo menciona, (Mackane, et al. 2017). Estándares han impulsado a las organizaciones a utilizar la energía de manera responsable, cumpliendo no solo con los modelos de rendimientos energéticos, sino con modelos de madurez energética basado en la NTC ISO 50001 que vincula los procesos y los criterios de integración del modelo de madurez que superan los requisitos de certificación (Jovanovic, 2016). 
Adicional a lo anteriormente expuesto se debe recordar que a través de la ley 1715 de 2014 se establece el marco legal y los instrumentos para la promoción del aprovechamiento de las fuentes no convencionales de energía, principalmente aquellas de carácter renovable, lo mismo que para el fomento de la inversión, investigación y desarrollo de tecnologías limpias para producción de energía, la eficiencia energética y la respuesta de la demanda, en el marco de la política energética nacional. Igualmente, tiene por objeto establecer líneas de acción para el cumplimento de compromisos asumidos por Colombia en materia de energías renovables, gestión eficiente de la energía y reducción de emisiones de gases de efecto invernadero, tales como aquellos adquiridos a través de la aprobación del estatuto de la Agencia Internacional de Energías Renovables (IRENA) mediante la Ley 1665 de 2013 (Pereira, 2017).

En la universidad de La Guajira sede Riohacha, existen datos históricos relacionados con el consumo de energía, suministrado por la oficina de recursos físicos de la universidad) en los cuales se logra evidenciar un aumento durante el periodo (2015-2016). Para el año 2015 el consumo promedio fue de $29.112 \mathrm{KW} / \mathrm{h}$ (Kilovatio hora), mientras que para el 2016 aumentó a $30.594 \mathrm{KW} / \mathrm{h}$, presentándose un incremento del $5 \%$ que sin duda alguna representa un impacto económico, social y ambiental a esta entidad. Se ha podido determinar mediante seguimiento y monitoreo el uso inadecuado de equipos eléctricos y electrónicos, que es promovido por docentes, estudiantes y funcionarios administrativos de la institución; se realizaron inspecciones en las horas en las que no se requiere su funcionamiento para el año 2016, de 77 aulas de clases disponibles, 39 aulas se encontraron con luminaria y ventiladores en funcionamiento sin la presencia de estudiante y docente, durante el horario de 12:30 pm y 1:29 pm; así también para el área administrativa se registraron 43 oficinas consumiendo energía, de las cuales 23 funcionaron en el horario de 12:00 pm y 2:59 pm.

La oficina de planeación de la Universidad de La Guajira, soporta datos de la población, donde se determina que el crecimiento poblacional es un factor determinante, ligado directamente al consumo de energía que año tras año cobra mayor relevancia, al observarse que para el año 2015 se consolidó una población de 12228 estudiantes y para el 2017 registró un aumento llegando a 14333 estudiantes, lo cual indica que se presentó un incremento en la población del 14,68\% a lo largo de dos años.

Es sin lugar a dudas, de acuerdo a lo que se logra evidenciar con relación al fenómeno objeto de estudio; una necesidad adoptar medidas que conlleven al uso y ahorro eficiente de la energía, con el objetivo de aprovechar al máximo el recurso energético y así mismo generar medidas de choque con el fin de impedir que continúe el aumento de los costos por el uso del servicio; ya que para el año 2015 la institución realizó pagos por concepto de energía por valor de \$189.701.800, y en el año 2016 por $\$ 263.808 .520$, generándose por este concepto un incremento del $28 \%$ en el valor pagado durante este periodo. En promedio la institución paga al año por el uso del servicio \$226.755.160, lo que representa un elevado costo para la institución educativa.

En conclusión, se ha logrado determinar que el consumo de energía eléctrica en la Universidad de La Guajira es elevado y una causa de este fenómeno es el uso irresponsable de equipos y maquinarias, por el descuido por parte de estudiantes, profesores y funcionarios de apagar las luces y equipos cuando salen de las instalaciones. Se afirma que estas dos áreas (bloques y laboratorios) son las de mayor influencia debido a que estas infraestructuras necesitan una climatización óptima para la efectividad de la prestación del servicio, sabiendo que las ofertas académicas que presentan mayor demanda estudiantil apoyan sus actividades en los laboratorios. Otro factor que incide sobre este fenómeno es el aumento de la infraestructura, actualmente estas áreas se encuentran activas e inciden directamente en las lecturas de consumo de energía eléctrica emanadas por el medidor que se encuentra en el área de los bloques.

\section{METODOLOGÍA}

El presente trabajo de investigación es de tipo descriptivo en referencia, a lo dicho por (Hernández, et al. 2014), se busca especificar las propiedades, las características y los perfiles de personas, grupos, comunidades, procesos, objetos o cualquier otro fenómeno que se someta a un análisis y es de diseño no experimental porque que se realiza sin manipular deliberadamente la variable. Las herramientas de recolección de datos fueron los registros de facturas de energía eléctrica, procesos y procedimientos de la organización e inventario de los equipos y maquinas.

La metodología se desarrolla mediante el modelo de gestión integral de la energía (MGIE) realizado por la universidad del atlántico y la universidad Autónoma de occidente entre los años 2006 y 2007, (Campos et al. 2008) el cual se adapta a los lineamientos de la NTC ISO 50001:2011, porque comprende actividades de implementación, operación y sostenimiento asociado al ciclo PHVA ( Planear, hacer, verificar, actuar) y se integra fácilmente a todos los niveles de la organización para el logro de la mejora continua del desempeño energético como se muestra en la Figura1 y Figura 2. 


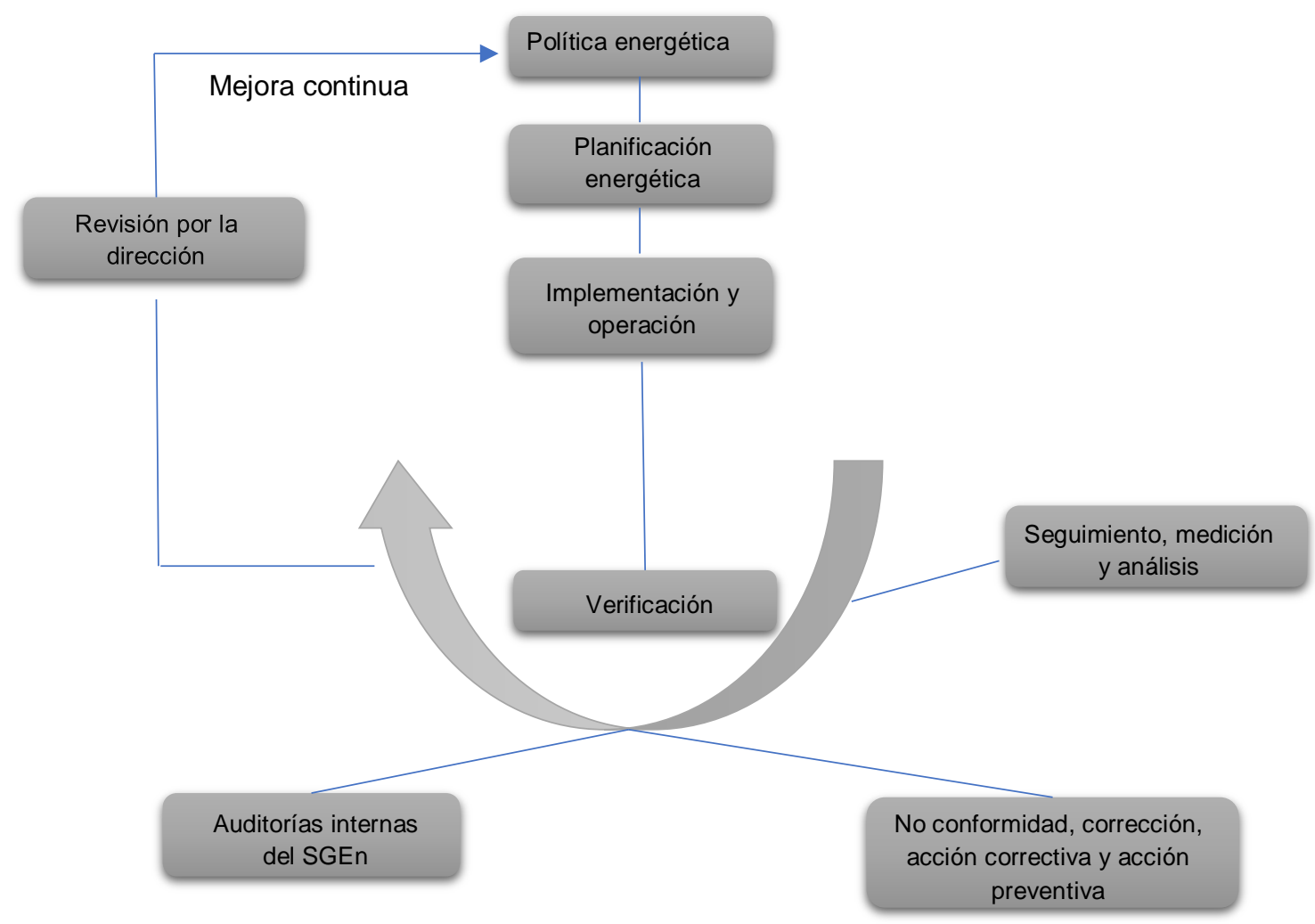

Fig. 1: Modelo de sistema de gestión de la energía

Etapa 3. Operación del sistema de Gestión integral de la energía.

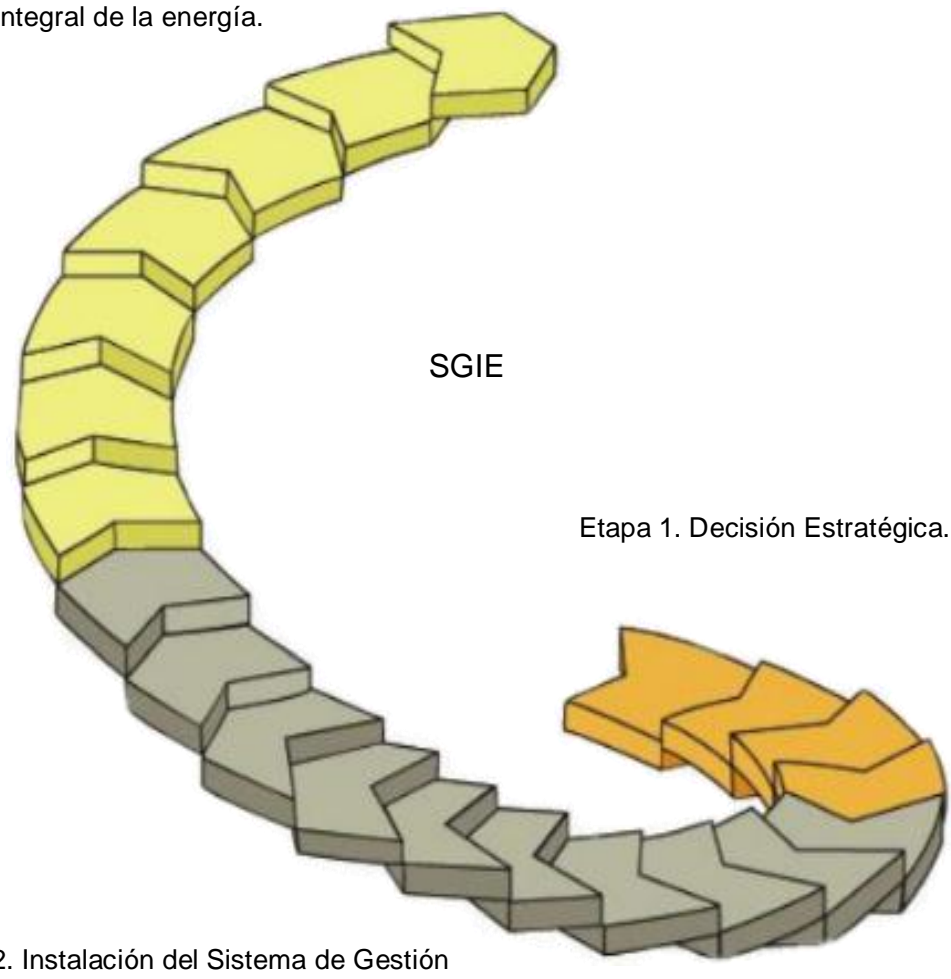

Etapa 2. Instalación del Sistema de Gestión Integral de la Energía

Fig. 2: Modelo de gestión integral de la energía (MGIE) NTC ISO 50001:2011 
Existe tres fases del MGIE, que sirven de preparación para la empresa que está decidida por certificarse o mejorar su desempeño energetico con la NTC ISO 50001, porque generalmente no cuentan con una cultura organizacional que sea eficiente a los procesos y procedimientos establecidos. El requisito 4.4 contempla la fase de planificación energética que integra las generalidades, revisión energética, línea de base, indicadores de desempeño, requisitos legales y otros requisitos, objetivos, metas, y planes de acción de la gestión energética. En la Tabla 1 se aprecia las actividades del MGIE, que son necesarias para obtener como producto final la disminución del consumo de energía eléctrica y bajos costos.

Este estudio desarrolla actividades que corresponden a la primera etapa del modelo de gestión integral de la energía (MGIE) con el fin de establecer la planificación del sistema de gestión de energía según lo especificado en la NTC ISO 50001:2011 y de esta forma generar un bosquejo que determine la viabilidad del sistema de gestión de energía de la universidad de La Guajira sede Riohacha. Para tal fin se consideran actividades propias de la planificación del sistema de gestión de energía, las establecidas en la etapa que involucra la decisión estratégica donde se definen los compromisos que asumen a nivel organizacional, con el objetivo de articular el diseño estructural de la institución con los requerimientos del sistema especificados anteriormente (Quispe et al. 2011).

Tabla 1. Ruta del modelo de gestión integral de la energía.

\begin{tabular}{|c|c|c|}
\hline Etapa 1. Decisión estratégica & Etapa 2. Instalación del SGIE & Etapa 3. Operación del SGIE \\
\hline \multirow[b]{2}{*}{ Caracterización de la empresa } & Establecimiento de los indicadores del sistema de gestión & Seguimiento y divulgación de indicadores \\
\hline & $\begin{array}{l}\text { Identificación de las variables de control por centros de costo y } \\
\text { áreas de gestión organizacional }\end{array}$ & $\begin{array}{l}\text { Seguimiento y evaluación de buenas } \\
\text { prácticas de operación, mantenimiento, } \\
\text { producción y coordinación }\end{array}$ \\
\hline \multirow[t]{3}{*}{ Compromiso de la alta dirección } & $\begin{array}{l}\text { Identificación de acciones correctivas, de control de eventos, } \\
\text { procedimientos operacionales y de gestión y proyectos } \\
\text { potenciales de uso racional de la energía (URE) en procesos }\end{array}$ & \multirow[t]{2}{*}{$\begin{array}{l}\text { Implementación de programas y } \\
\text { proyectos de mejora }\end{array}$} \\
\hline & Definición de los sistemas de monitoreo & \\
\hline & Diagnostico energético & Chequeos de gerencia \\
\hline \multirow{3}{*}{$\begin{array}{l}\text { Alineación de la estructura de la } \\
\text { empresa hacia el uso racional de } \\
\text { la energía }\end{array}$} & $\begin{array}{l}\text { Identificación de oportunidades, soluciones y medidas de uso } \\
\text { eficiente de la energía }\end{array}$ & \multirow{2}{*}{$\begin{array}{l}\text { Implementación del plan de } \\
\text { entrenamiento y evaluación del personal }\end{array}$} \\
\hline & Actualización y validación de la gestión organizacional del SGIE & \\
\hline & Preparación del personal & \multirow{2}{*}{ Ajustes del sistema de gestión } \\
\hline \multirow{3}{*}{$\begin{array}{l}\text { Validación y actualización de la } \\
\text { estructura organizativa actual de } \\
\text { los procesos }\end{array}$} & Auditoría interna al SGIE & \\
\hline & Implementación y seguimiento del SGIE & \multirow{2}{*}{ Evaluación de resultados } \\
\hline & Documentación del SGIE & \\
\hline
\end{tabular}

\section{Decisión estratégica}

Tiene como objetivo generar el compromiso de la alta dirección de la empresa para la asignación de recursos e insertar en su gestión organizacional el SGIE. En esta etapa se identifica el estado actual de la empresa, las metas globales y los impactos en la productividad, el medio ambiente, la utilidad, los gastos operacionales y el rendimiento, entre otras. Las actividades que conforman esta etapa son las siguientes como se detalla en la Tabla 2:

Tabla 2. Actividades de la etapa de decisión estratégica.

\begin{tabular}{|l|l|}
\hline \multicolumn{1}{|c|}{ Actividades } & \multicolumn{1}{c|}{ Definición } \\
\hline $\begin{array}{l}\text { Caracterización de la } \\
\text { empresa }\end{array}$ & $\begin{array}{l}\text { Se realiza una identificación de los procesos de la empresa y al uso eficiente de la energía, que consiste en la } \\
\text { aplicación de herramientas de caracterización para la determinación del potencial de ahorro total por reducción de la } \\
\text { variabilidad operacional, de la planeación de la producción y de la mejora de la capacidad técnica organizativa de la } \\
\text { empresa para administrar la energía en forma eficiente como lo expresa (Prias et al. 2013). }\end{array}$ \\
\hline $\begin{array}{l}\text { Compromiso de la alta } \\
\text { dirección }\end{array}$ & $\begin{array}{l}\text { Con base al diagnóstico inicial de la empresa se determinan los requisitos legales propuestos en la NTC ISO } \\
50001: 2011, \text { también se establecen los compromisos de tiempos, tareas y de reducción de costos energéticos } \\
\text { durante la instalación y operación del sistema. Específicamente se deben definir las formas en que se garantizaran } \\
\text { los requisitos de instalación y operación; como también precisar los objetivos y alcances del SGIE, según (Prías et } \\
\text { al. 2013) }\end{array}$ \\
\hline $\begin{array}{l}\text { Alineación de la estructura } \\
\text { de la empresa hacia el uso } \\
\text { racional de la energía }\end{array}$ & $\begin{array}{l}\text { Para la verificación del desempeño de las estrategias, planes y programas que conlleven a la mejora de la eficiencia } \\
\text { energética se hace necesario realizar un seguimiento que le compete a la alta dirección. En este sentido, la NTC ISO } \\
50001 \text { integra prácticas de gestión, el papel de la organización es fundamental para que pueda existir una } \\
\text { implementación exitosa, además, el apoyo de la administración es importante para garantizar procesos de } \\
\text { planificación e implementación, asignación de recursos, establece las funciones y responsabilidades del equipo de } \\
\text { energía para generar una cultura (Fuchs et al. 2020). }\end{array}$ \\
\hline $\begin{array}{l}\text { Validación y actualización de } \\
\text { la estructura organizativa } \\
\text { actual de los procesos }\end{array}$ & $\begin{array}{l}\text { De acuerdo a la NTC ISO 50001:2011 esta etapa corresponde a la planeación donde se identifica con que cuenta la } \\
\text { empresa para realizar un diseño de un Sistema de Gestión Integral de la Energía, SGIE, acorde con sus necesidades. }\end{array}$ \\
\hline
\end{tabular}




\section{RESULTADOS Y DISCUSIÓN}

En la revisión de la funcionalidad de los procesos que conforman la organización y afectan el desempeño energético como lo establece la NTC ISO 50001 en el requisito número 4.4, se tiene claridad del comportamiento del consumo en la Universidad de la Guajira y de este modo se logró establecer estrategias para mitigar el impacto.

\section{Estructura de procesos de la Institución}

El sistema de gestión de calidad básicamente se encuentra estructurado bajo 4 tipos de procesos de gran envergadura, estratégicos, misionales, apoyo y evaluación que se despliegan de la estructura organizacional, Tabla 3. Para cada tipo de proceso dentro del sistema de gestión de calidad de la universidad de la Guajira, existen unas rutas o procedimientos que son de alta aplicabilidad para cada dependencia, para así cumplir con los objetivos y metas establecidas por esta entidad.

Tabla 3. Procesos y funcionalidad (Fuente: Adoptado del mapa de procesos de la universidad de La Guajira).

\begin{tabular}{|l|l|l|}
\hline Tipo de proceso & Proceso & Funcionalidad del proceso \\
\hline Estratégico & $\begin{array}{l}\text { Planeación institucional, sistema integrado de gestión, } \\
\text { comunicación institucional, aseguramiento de la calidad. }\end{array}$ & Administrativo \\
\hline Misional & Docencia, investigación y proyección social & Laboratorio y trabajo de campo \\
\hline Apoyo & $\begin{array}{l}\text { Gestión del talento humano, documental, laboratorios, } \\
\text { admisiones y control de registro, bienestar universitario, } \\
\text { jurídica, tecnológica e infraestructura académica, } \\
\text { internacionalización, administrativa y financiera. }\end{array}$ & $\begin{array}{l}\text { Administrativo- mantenimiento y } \\
\text { control de equipos. }\end{array}$ \\
\hline Evaluación & Control de gestión y evaluación de Resultados & Administrativo \\
\hline
\end{tabular}

Panorama actual del consumo de la energía eléctrica

En este estudio, se tomaron como base los consumos de energía registrados en las facturas de los años 2015, 2016 y primer semestre del 2017, debido a que antes de implementar el sistema de gestión de calidad no se contaba con un control adecuado de la información. Adicionalmente se identificaron alrededor de la universidad de La Guajira sede Riohacha 5 contadores que monitorean por sectores el consumo de la energía dentro de la infraestructura.

\section{Áreas con mayor consumo de energía eléctrica}

Con el fin de determinar las áreas que generan mayor consumo de energía eléctrica en la institución, se establecieron los consumos de energía correspondientes a cada bloque, tomando como base los años 2015, 2016 y 2017, para la representación de los datos se utilizó un diagrama de Pareto (Figura 3) el cual permitió la agrupación de una manera satisfactoria de todos los datos.

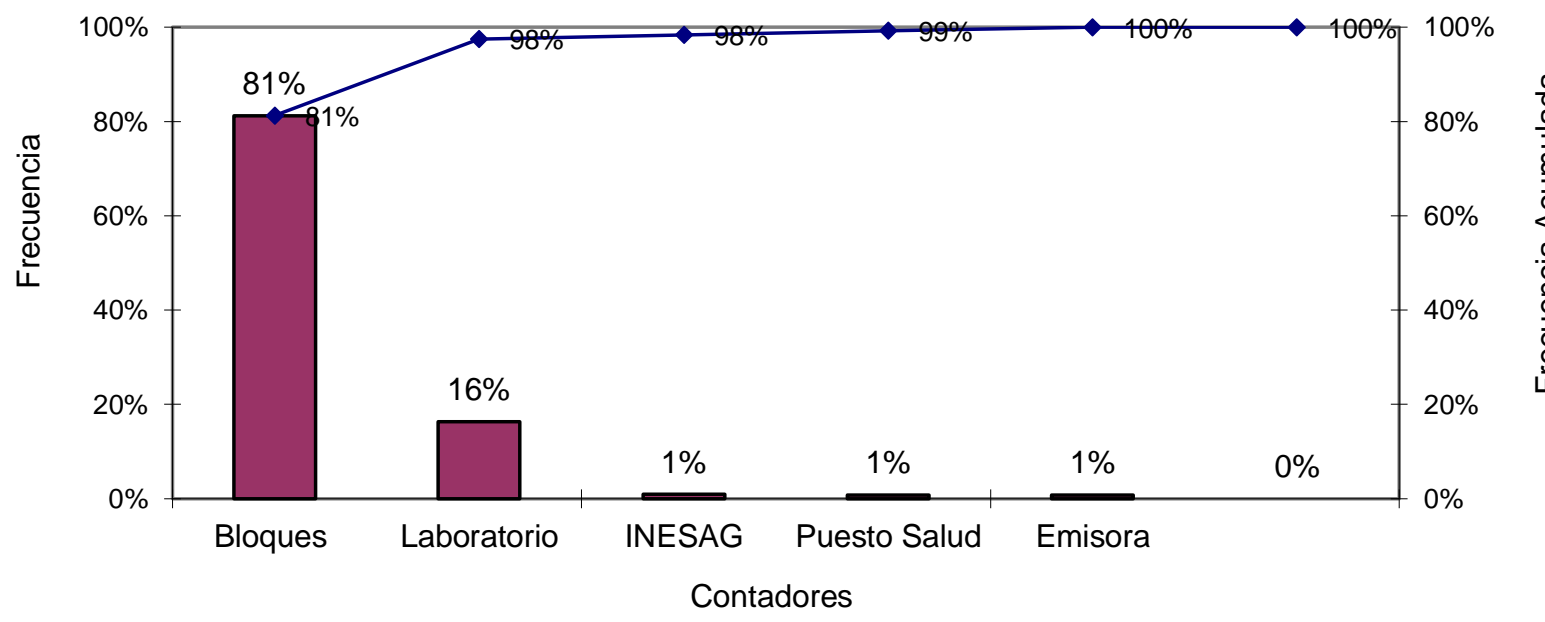

Fig. 3: Diagrama de Pareto para contadores 
Como se observa en el Figura 3, las áreas que demandan mayor consumo de energía eléctrica son los bloques y los laboratorios, uno de los factores que inciden sobre este fenómeno es la cantidad de aires acondicionados disponibles, en donde se evidencia que para el año 2017 se registraron 149 aires acondicionados activos en total, de las cuales 105 equivalentes al 70,46\% del total pertenecen al área de los bloques, y 30 equivalentes al 20,13\% pertenecen a los laboratorios. Se afirma que estas dos áreas son las de mayor influencia debido a que estas infraestructuras son necesarias y necesitan una climatización óptima para la efectividad de la prestación del servicio, sabiendo que las ofertas académicas que presentan mayor demanda estudiantil apoyan sus actividades en los laboratorios. (Valdés, et al. 2020) recomienda la implementación de paneles fotovoltaicos como fuente de generación de energía eléctrica para las viviendas de Arica, se considera que es un proyecto rentable en términos económicos, ambiental, y legal, en este sentido a la universidad de La Guajira, le serviría realizar instalación de fuentes No convencionales por las condiciones climáticas con las que cuenta.

Otro factor que incide sobre este fenómeno es el aumento de la infraestructura, en donde se evidencia que para el año 2013 no contaba con las infraestructuras y el bloque VI, actualmente estas áreas se encuentran activas e inciden directamente en las lecturas de consumo de energía eléctrica emanadas por el medidor que se encuentra en el área de los bloques. Además, se debe resaltar que aparte de las actividades académicas, también el sector administrativo juega un papel importante en el consumo de energía en los bloques, de acuerdo a la composición estructural y organizacional de la universidad, en cada bloque existe una facultad que posee una oficina de decanatura y cubículos para asesorías por parte del personal docente, en donde se evidencia que el aire acondicionado proporciona ventilación a todas las oficinas sin importar si todo el personal está laborando, del mismo modo ocurre con el alumbrado.

\section{Análisis del consumo de energía en los bloques mes a mes}

Con el fin de contar con mayor precisión al profundizar en la identificación de los elementos necesarios de planificar un sistema de gestión de energía acorde a lo establecido en la NTC 50001:2011, se realizó la consolidación de las cifras correspondientes al consumo de energía eléctrica que se reporta en cada uno de los bloques, esto con una frecuencia de mes a mes entre los años 2015,2016 y 2017, información que se relaciona en la Tabla 4 y en la Tabla 5, de la misma forma se registró al detalle la tendencia del consumo de la energía en los años 2015-2016 (Figura 4) y 2017 (Figura 5) con la finalidad de establecer diferencias en consumo mes a mes.

Tabla 4. Consumo energético y costo mensual en los bloques universitarios años 2015 y 2016

\begin{tabular}{|c|c|c|c|c|c|c|}
\hline \multicolumn{9}{|c|}{2015} & \multicolumn{3}{c|}{ Bloques } \\
\hline Mes & Consumo KWH & Tarifa (\$) & $\begin{array}{c}\text { Montos cancelados } \\
\text { (aprox) }\end{array}$ & Consumo KWH & Tarifa (\$) & $\begin{array}{c}\text { Montos cancelados } \\
\text { (aprox) }\end{array}$ \\
\hline Enero & 39670 & $\$ 282,41$ & $\$ 11.203 .205$ & 42600 & $\$ 380,98$ & $\$ 16.229 .748$ \\
\hline Febrero & 77670 & $\$ 283,65$ & $\$ 22.031 .096$ & 85360 & $\$ 387,64$ & $\$ 33.088 .950$ \\
\hline Marzo & 135780 & $\$ 285,06$ & $\$ 38.705 .447$ & 155760 & $\$ 394,81$ & $\$ 61.495 .606$ \\
\hline Abril & 112670 & $\$ 328,73$ & $\$ 37.038 .009$ & 127600 & $\$ 376,98$ & $\$ 48.102 .648$ \\
\hline Mayo & 120450 & $\$ 316,42$ & $\$ 38.112 .789$ & 148720 & $\$ 364,14$ & $\$ 54.154 .901$ \\
\hline Junio & 125230 & $\$ 321,48$ & $\$ 40.258 .940$ & 128040 & $\$ 344,62$ & $\$ 44.125 .145$ \\
\hline Julio & 153210 & $\$ 320,63$ & $\$ 49.123 .722$ & 148720 & $\$ 352,54$ & $\$ 52.429 .749$ \\
\hline Agosto & 118360 & $\$ 323,71$ & $\$ 38.314 .316$ & 128040 & $\$ 348,57$ & $\$ 44.630 .903$ \\
\hline Septiembre & 130240 & $\$ 328,03$ & $\$ 42.722 .627$ & 138160 & $\$ 358,01$ & $\$ 49.462 .662$ \\
\hline Octubre & 171160 & $\$ 354,48$ & $\$ 60.672 .797$ & 109560 & $\$ 350,23$ & $\$ 38.371 .199$ \\
\hline Noviembre & 154880 & $\$ 359,68$ & $\$ 55.707 .238$ & 164560 & $\$ 362,23$ & $\$ 59.608 .569$ \\
\hline Diciembre & 135960 & $\$ 360,21$ & $\$ 48.974 .152$ & 90640 & $\$ 364,51$ & $\$ 33.039 .186$ \\
\hline
\end{tabular}




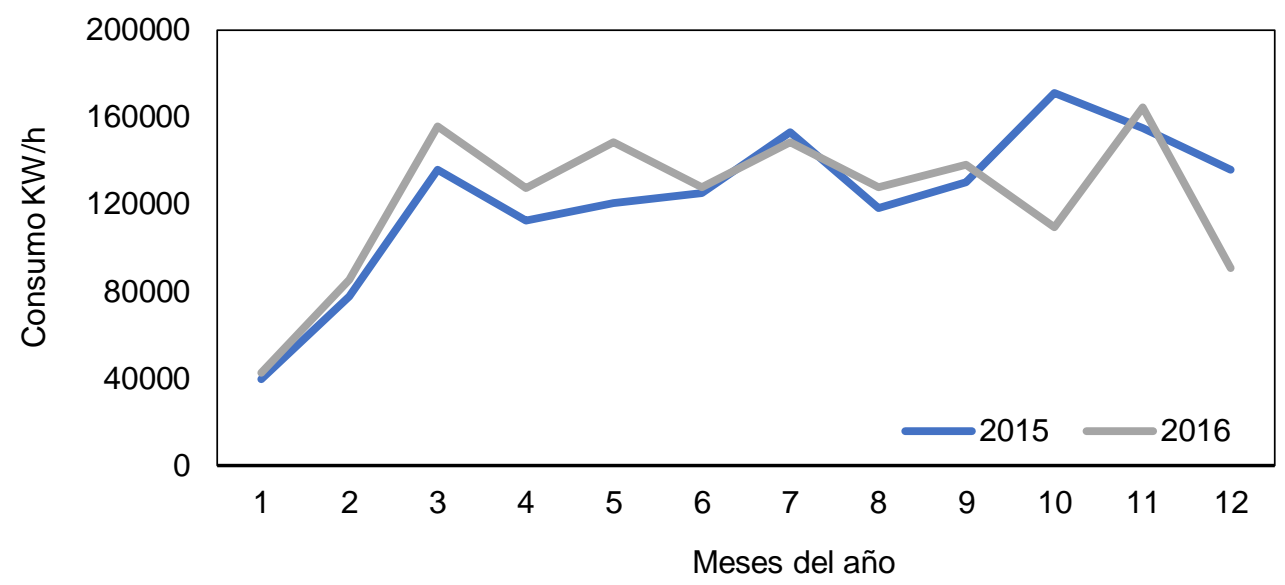

Fig. 4: Comportamiento del consumo energético en los bloques universitarios años 2015-2016

Con respecto a lo observado en la Figura 4 se puede inferir que en el año 2016 hubo variaciones en los consumos que son más elevados que para el año 2015, sin embargo, termina evidenciándose un menor consumo finalizando el año en comparación con el año anterior. También se observa que existe gran similitud en el comportamiento de consumo de energía eléctrica durante los primeros semestres de cada año, aclarando que para el primer semestre del año 2016 se presentó un mayor consumo de energía al del año anterior, considerando que para el mismo año existió un incremento en la oferta de programas académicos, lo cual provocó la inclusión de mayor número de estudiantes a la institución. Durante los meses de abril y junio de los dos años se observan disminuciones en el consumo de la energía, dado que para esas fechas inician las temporadas vacacionales y por tal motivo se suspenden la mayor parte de las actividades que se desarrollan en la institución. En cuanto al comportamiento del consumo en el año 2016, específicamente en el segundo semestre, se logró percibir que desde el mes de octubre en adelante se presentó un descenso en el consumo de la energía eléctrica, provocado por un cese de actividades académicas debido a decisiones administrativas, y por ende la tendencia de consumo para este periodo fue menor.

Tabla 5. Consumo energético y costo mensual en los bloques universitarios año 2017

\begin{tabular}{|c|c|c|c|}
\hline \multicolumn{4}{|c|}{ Bloques 2017} \\
\hline Mes & Consumo kwh & Tarifa $(\$)$ & $\begin{array}{c}\text { Montos cancelados } \\
\text { (aproximadamente) }\end{array}$ \\
\hline Enero & 135520 & $\$ 320,26$ & $\$ 43.401 .635$ \\
\hline Febrero & 47080 & $\$ 334,09$ & $\$ 15.728 .957$ \\
\hline Marzo & 80520 & $\$ 359,89$ & $\$ 28.978 .343$ \\
\hline Abril & 128040 & $\$ 356,70$ & $\$ 45.671 .868$ \\
\hline Mayo & 127160 & $\$ 339,68$ & $\$ 48.193 .709$ \\
\hline Junio & 150920 & $\$ 321,89$ & \\
\hline
\end{tabular}

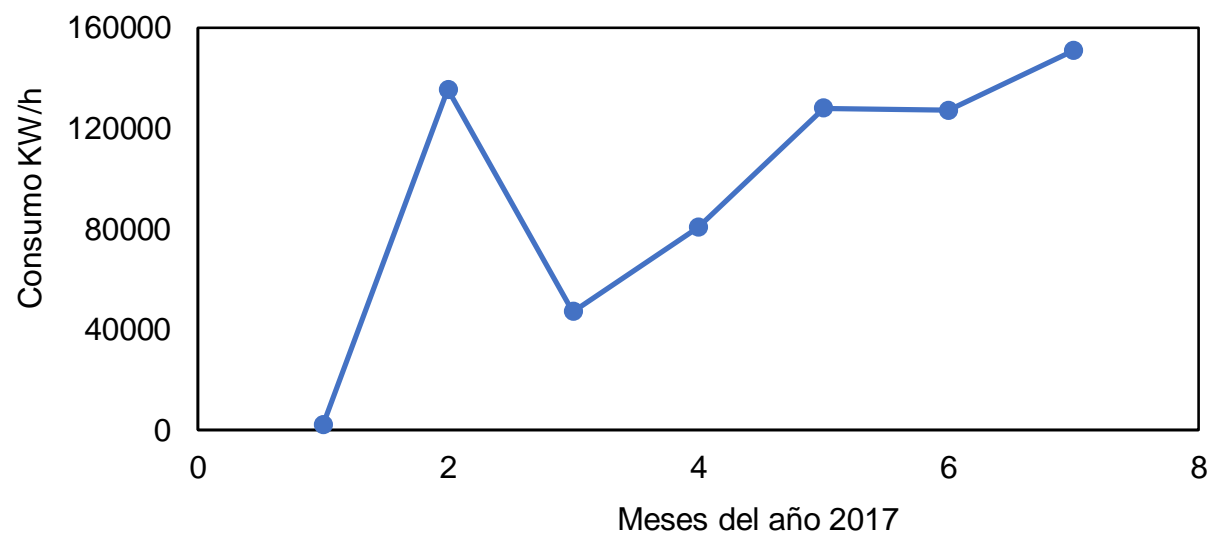

Fig. 5: Comportamiento del consumo energético en los bloques universitarios año 2017 
Con respecto a la tendencia, se evidencia un aumento de consumo teniendo en cuenta que para este periodo académico entro en funcionamiento a plenitud el bloque VI, según información suministrada por la oficina de recursos físicos, esta cuenta con una capacidad de 1770 estudiantes, 35 aulas de clase, ocho (8) aulas múltiples y salas docentes, lo cual es de gran relevancia para el consumo de la energía en esta institución, debido a la intensidad de uso que la infraestructura presenta diariamente.

\section{Análisis del consumo de energía total}

Para este caso se consolidó el consumo total de energía de acuerdo a cada bloque por año, de la misma forma se detalló la tendencia del consumo total de la energía en los años 2015,2016 y 2017 (Figura 6) y (Tabla 6) con la finalidad analizar el comportamiento de consumo manifestado por las diferentes secciones en los años anteriormente mencionados.

Tabla 6. Consumo de energía eléctrica total.

\begin{tabular}{|l|l|l|l|}
\hline \multicolumn{1}{|c|}{ Contador } & \multicolumn{3}{c|}{ Consumo en KWH } \\
\hline & 2015 & 2016 & 2017 \\
\hline Bloques & 1475280 & 1467760 & 669240 \\
\hline Puesto de Salud & 16240 & 13440 & 6880 \\
\hline INESAG & 15836 & 16063 & 7213 \\
\hline Laboratorio & 239360 & 328680 & 154440 \\
\hline Emisora & 14021 & 9723 & 10318 \\
\hline
\end{tabular}

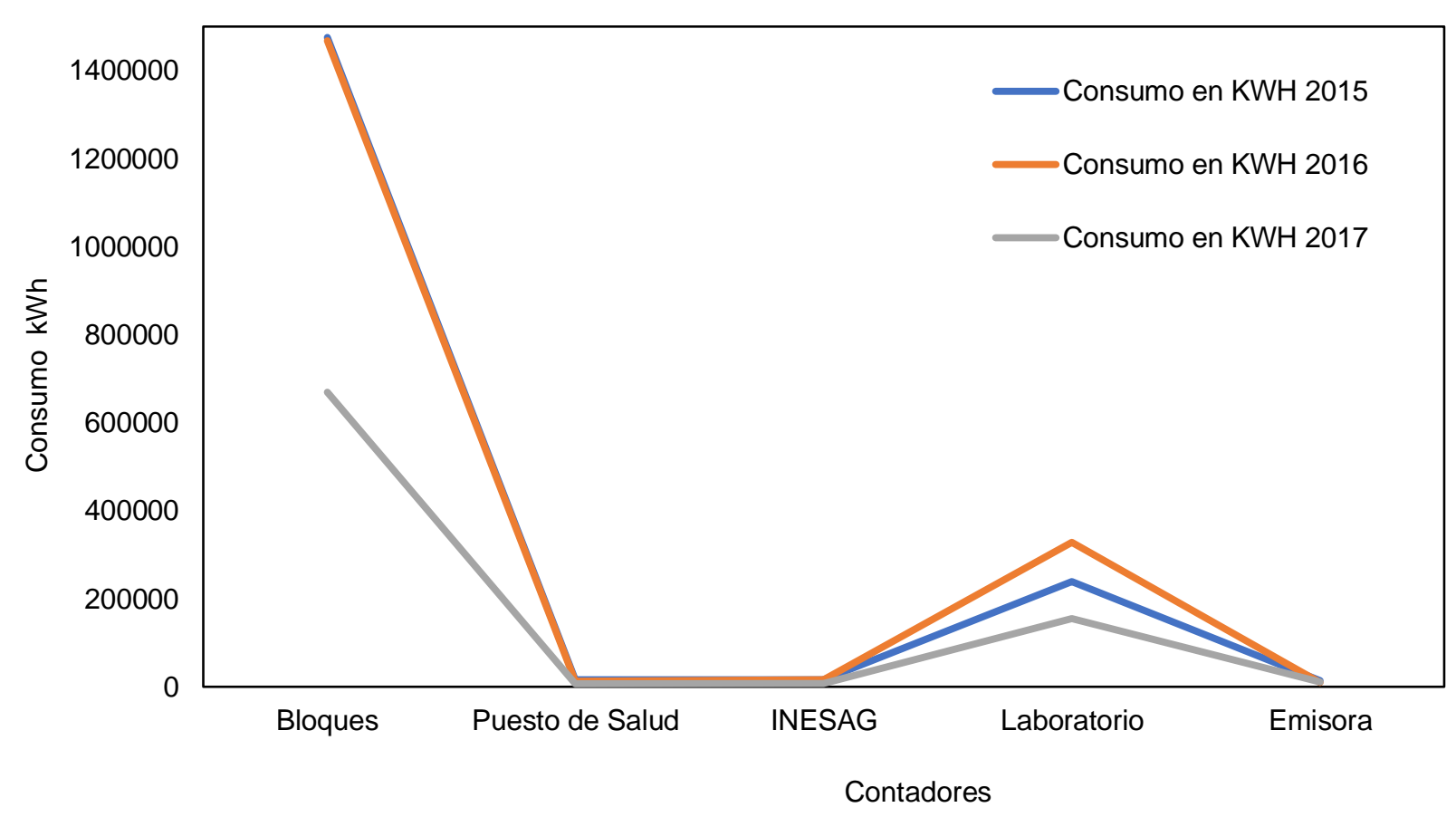

Fig. 6: Comportamiento de Consumo de energía eléctrica total de la Universidad de la Guajira en los años 2015,2016 y 2017.

Al tener claridad acerca del diagnóstico energético de la institución se logra identificar que, debido a la magnitud de la infraestructura y dado que cada año presenta novedades en cuanto a sus dimensiones, el consumo de energía eléctrica muestra un comportamiento ascendente, también se logra observar que solo se manifiestan disminuciones en el consumo de energía eléctrica cuando existen causas externas como el cese de actividades académicas ocasionadas por vacaciones o por otras causas. De acuerdo a lo expresado en la Figura 6, se observa un mayor consumo para el año 2016 por encima del 2017, es importante resaltar que los datos del año 2017 solo se encuentran registrados hasta el primer semestre del mismo. 
Consumo per cápita de la población universitaria, años 2015 al 2017.

Se determinó la población de acuerdo al tipo de personal vinculado a la universidad de La Guajira, como se observa en la (Tabla 7) y (Figura 7), además se estableció el consumo per cápita para cada año 2015, 2016 y 2017.

Tabla 7 Población y consumo per cápita de la Universidad de La Guajira.

\begin{tabular}{|l|c|c|c|}
\hline Personal & 2015 & 2016 & 2017 \\
\hline Estudiantes & 10895 & 11902 & 13000 \\
\hline Funcionarios y docentes de planta & 173 & 173 & 930 \\
\hline Catedráticos & 930 & 930 & 192 \\
\hline Ocasionales & 192 & 192 & 13 \\
\hline Operarios de fotocopiadora & 13 & 13 & 25 \\
\hline Operarios de cafetería & 25 & 13253 \\
\hline \multicolumn{2}{|c|}{ TOTAL Consumo Per Cápita (consumo de energía kWh/total de la población) } \\
\hline \multicolumn{2}{|c|}{1.760 .737} & 1.835 .666 & 848.091 \\
\hline Consumo total de energía & 142,9 & 138,7 & 59,17 \\
\hline Consumo per cápita & & & 1433 \\
\hline
\end{tabular}

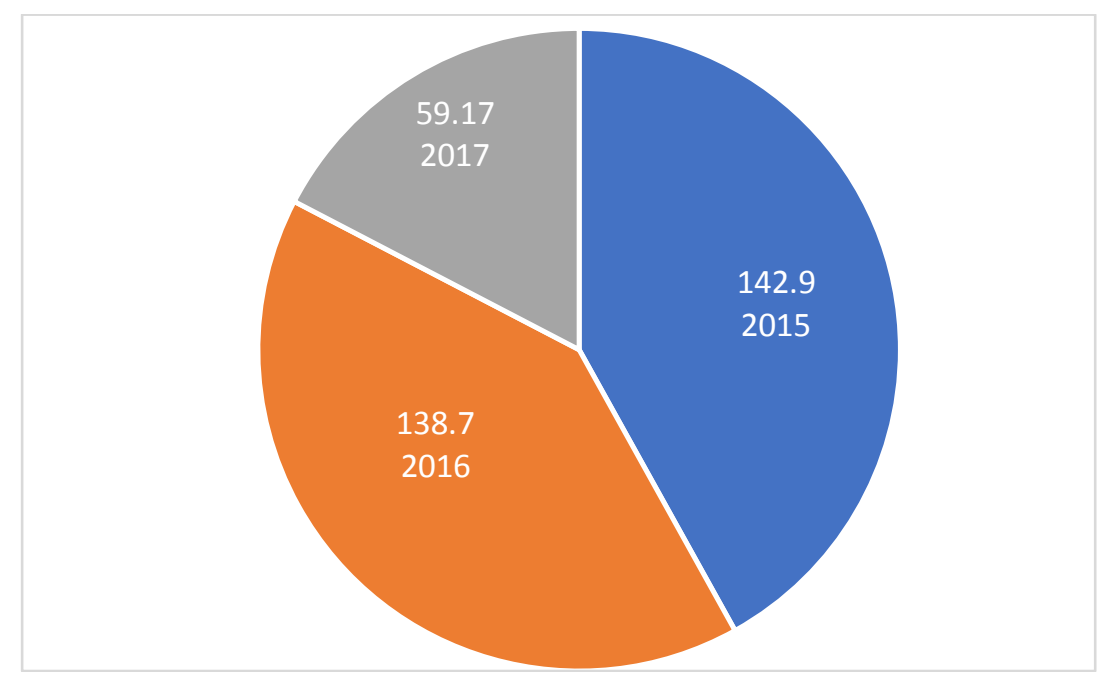

Fig. 7: variación del consumo de energía eléctrica per cápita $(2015,2016,2017)$

Todo esto nos permite observar que la universidad se ha desarrollado de manera significativa, y al aumentar la población, por ende, se ampliará el consumo de la energía eléctrica. Con respecto al consumo per cápita, se observa en la (Tabla 7) y (Figura 7), que para el año 2015 fue de 142,9 Kwh/hab, mientras para el año 2016 se redujo en un $3 \%$, siendo para ese año de 138,7 Kwh/hab; en el año 2017 se tomaron datos del primer periodo académico, lo que representa el consumo per cápita de 59,17 Kw/hab. Uno de los factores que incidieron sobre este descenso fue la sequía, por lo tanto, los entes gubernamentales tomaron la iniciativa de establecer soluciones oportunas para evitar el desperdicio de agua. En este sentido, se evidencia que la toma de conciencia promovida por la empresa prestadora del servicio que realizaba con frecuencia razonamientos de energía e incentivaba al ahorro de energía por medio de descuentos reflejados en el pago del servicio a través de la campaña llamada "apagar paga" arrojó buenos resultados.

\section{Soluciones para disminuir el consumo de energía}

Se propone la implementación de un programa de ahorro y uso eficiente de la energía (Tabla 8 y 9), y adicionalmente un modelo de seguimiento con la finalidad de disminuir los costos excesivos que surgen del manejo inadecuado de la energía eléctrica. 
Tabla 8. Programa para el uso eficiente y ahorro de energía eléctrica

Programa para el uso eficiente y ahorro de energía eléctrica-PUEAEE

Objetivo: establecer alternativas mediante la implementación de estrategias o actividades que contribuyan al uso racional y eficiente de energía, con el fin de obtener buenos resultados de un consumo responsable.

Metas:

Sensibilizar a los empleados, estudiantes y comunidad en general sobre la necesidad de asumir comportamientos responsables en el uso de energía, con el fin de eliminar pérdidas innecesarias.

Aportar criterios y alternativas en cuanto al uso y ahorro de energía.

Reducir el consumo de energía eléctrica y adoptar medidas de ahorro establecidos por la ley.

\begin{tabular}{|c|c|}
\hline \multicolumn{2}{|l|}{ Medidas de prevención } \\
\hline Medidas y/o actividades & Tiempo de ejecución \\
\hline Apagar y desconectar luces y electrodomésticos que no se usen. & Permanente \\
\hline $\begin{array}{l}\text { Cerrar las puertas cuando hay aire acondicionado en funcionamiento, para tratar de } \\
\text { conservar la temperatura y no forzar el sistema. }\end{array}$ & Permanente \\
\hline Instalar artefactos y accesorios electrónicos con elevada eficiencia energética. & 2 meses \\
\hline \multicolumn{2}{|l|}{ Medidas de mitigación } \\
\hline Medidas y/o actividades & Tiempo de ejecución \\
\hline $\begin{array}{l}\text { Realizar un inventario de los artefactos y accesorios electrónicos. Para ello se debe usar } \\
\text { un formato de registro de artefactos y accesorios electrónicos. }\end{array}$ & Permanente \\
\hline Realizar un diagnóstico sobre el consumo de energía eléctrica y otras fuentes de energía. & Permanente \\
\hline $\begin{array}{l}\text { Realizar el registro de fugas energéticas y daños eléctricos (daño de equipos y accesorios) } \\
\text { mediante el formato periódico de detección de fugas energéticas y daños eléctricos. }\end{array}$ & Permanente \\
\hline \multicolumn{2}{|l|}{ Buenas prácticas ambientales } \\
\hline Buenas prácticas & Metodología de implementación \\
\hline $\begin{array}{l}\text { Capacitar a los empleados (docentes, funcionarios) sobre el uso eficiente y ahorro de } \\
\text { energía eléctrica, y el uso adecuado de los elementos electrónicos. }\end{array}$ & $\begin{array}{l}\text { Dictar capacitaciones a los empleados sobre el } \\
\text { programa para el uso eficiente y ahorro de } \\
\text { energía eléctrica- pueaee. }\end{array}$ \\
\hline Incentivar la participación de todos los usuarios en el uso eficiente de la energía eléctrica. & $\begin{array}{l}\text { Sensibilizar a los usuarios en la importancia del } \\
\text { uso eficiente de energía eléctrica. }\end{array}$ \\
\hline $\begin{array}{l}\text { Desarrollar modelos de comunicación e información para motivar a la participación y } \\
\text { colaboración de las personas en el buen uso de la energía eléctrica. }\end{array}$ & $\begin{array}{l}\text { Usar afiches, volantes y tableros informativos } \\
\text { donde se explique la importancia del uso } \\
\text { eficiente y ahorro de energía eléctrica. Estos } \\
\text { deben ubicarse en puntos de flujo de personal. }\end{array}$ \\
\hline Aprovechar la luz solar mediante el uso de paneles solares y la biomasa térmica. & $\begin{array}{l}\text { Implementar paneles solares que ayuden al } \\
\text { aprovechamiento de la energía solar, además } \\
\text { de la identificación de la biomasa térmica } \\
\text { gracias al aprovechamiento de los residuos } \\
\text { producidos internamente en la universidad. }\end{array}$ \\
\hline
\end{tabular}

\section{CONCLUSIONES}

Con el desarrollo de la presente investigación se ha logrado determinar: 1) que el consumo de energía eléctrica en la universidad de La Guajira es elevado y una causa de este fenómeno es el uso irresponsable de equipos y maquinarias, por el descuido por parte de estudiantes, profesores y funcionarios de no apagar las luces y equipos cuando salen de las instalaciones; 2) Las áreas con mayor influencia son los bloques y laboratorios, uno de los factores que inciden sobre este fenómeno es la cantidad de aires acondicionados disponibles y mal utilizados las dos áreas son de actividad académica y tiene mayor demanda estudiantil, además se ha tenido un crecimiento en la infraestructura y se encuentran activas; 3) el consumo de energía eléctrica muestra un comportamiento ascendente, solo se manifiestan disminuciones cuando existen causas externas como el cese de actividades académicas ocasionadas por vacaciones; 4) por tal motivo, como solución a la problemática se considera necesaria la implementación de un programa de ahorro y uso eficiente de la energía, adicionalmente un modelo de seguimiento con la finalidad de monitorear todas las acciones encaminadas al mejoramiento energético. 


\section{REFERENCIAS}

Arévalo, J., y Flórez, E.N. Diagnóstico del consumo de la energía eléctrica en las instalaciones de los laboratorios de la universidad Francisco de Paula Santander,asociación Colombiana de facultades (Acofi). ISBN: 978-958-680-079-2, 242 -278 (2016).

Bassani, M.L., y Osorio, R. Environmental protection as an indirect effect of the ISO 50001 energy management system. http://doi.org/10.5102/rdi.v14i3.4977, Rev. Centro universitario de Brasilia, 14(3), 106-120(2017).

Cabrera, P., Lund, H., y otros 2 autores. The matlab toolbox for energy plan: A tool to extend energy planning studies. http://doi.org/10.1016/j.scico.2020.102405 ,Rev. Science of computer programming,191,102- 405, (2020).

Campos, J.C., y Lora, E.D., y otros cuatro autores. Modelo de gestión energética para el sector productivo nacional. ISSN: 1692-8261, Rev. Prospectiva, 6 (1), 23-27(2008).

Deveci, K., y Güler, Ö. A cmopso based multi-objective optimization of renewable energy planning: Case of Turkey, http://doi.org/10.1016/j.renene.2020.03.033, Rev. Renewable energy,155, 578 - 590 (2020).

Fuchs, H., Aghajanzadeh, P., y otro autor. Identification of drivers, benefits, and challenges of ISO 50001 through case study content analysis. http://doi.org/10.1016/j.enpol.2020.111443, Rev. Energy policy,142,111443 (2020).

García, R.A., Flores, E., y Rodríguez, M. Application of the procedure of the NTC ISO 50001: 2011 standard for energy planning in the ceramic sector of a company. http://doi:10.15446/dyna.v86n209.70915, Rev. Universidad nacional de Colombia, 86 (209), 113-119, (2019).

Hernández, R., Fernández, C; y Baptista, M.P y otros dos autores. Metodología de la investigación. D.F. México. Editorial: McGrawHill Educación, 6ªedi. (2014).

ISO 50001. Sistemas de gestión de la energía, requisitos con orientaciones para su uso. Bogota Colombia, Instituto colombiano de normas técnicas y certificación Icontec (2011-2018).

Jovanovic, B., y Filipovic, J. ISO 50001 standard-based energy management maturity model e proposal and validation in industry. http://doi.org/10.1016/j.jclepro.2015.10.023, Rev. Cleaner production, 112 parte 4, 2744-2755, (2016).

Krog, L., y Sperling, K. A comprehensive framework for strategic energy planning based on Danish and international insights. http://doi.org/10.1016/j.esr.2019.02.005, Rev. Energy strategy, 24, 83 - 93, (2019).

Mackane, A., Therkelsen, P., y otros dieciséis autores. Predicting the quantifiable impacts of ISO 50001 on climate change mitigation, http://doi.org/10.1016/j.enpol.2017.04.049, Rev.Energy policy, 107, 278- 288, (2017).

Pereira, M.J. Tratamiento jurídico de las energias renovables en Colombia: ahorro energético, eficiencia energética y uso racional de la energía., Rev. Jurídica MArio Alario D’Filippo, 9(17), 43-68, (2017).

Prías, O.F., y Campos,J.C., y otros dos autores. implementación de un sistema de gestión de la energía guía con base en la norma iso 50001, 1ㄹ Ed; sistema de gestión integral de la energía, Bogota D.C.,(2013).

Poveda, P.P., García, J.C., y otros dos autores. An integral, risk and energy approach in management system. Analysis of ISO 50001: 2018, http://doi.org/10.18687/LACCEI2019.1.1.231, Rev. Latin american and caribbean consortium of engineering institutions, 152362, 2019.

Quispe, E. C., Castrillon, R. P y otros dos autores., El modelo de gestión energética Colombiano: desarrollo, experiencias y resultados de aplicación y perspectivas futuras de desarrollo, Conicca, IX congreso nacional y IV internacional de ciencia y tecnología del carbón y combustibles alternativos, Santiago de Cali, (2011).

Robles, C.A., Taborda, J.A y Ospino, A.J. Procedimiento para la Selección de Criterios en la Planificación Energética de Zonas Rurales Colombianas. http://dx.doi.org/10.4067/S0718-07642018000300071, Rev. Información tecnológica, 29(3), (2018)

Solangi, Y. A., Tan, Q., y otros dos autores. Evaluating the strategies for sustainable energy planning in Pakistan: An integrated SWOT-AHP and Fuzzy-TOPSIS approach. http://doi.org/10.1016/j.jclepro.2019.117655, Rev.Cleaner production, 236,117665, (2019).

Valdés, G.D, Rodríguez, E.R., y otros dos autores; Estudio de viabilidad de sistemas fotovoltaicos como fuentes de energía distribuida en la ciudad de Arica, Chile. http://dx.doi.org/10.4067/S0718-07642020000300249, Rev, información tecnológica, 31(3), (2020)

Wang, N., Heijnen, P.W., y Imhof, P.J. A multi-actor perspective on multi-objective regional energy system planning, http://doi.org/10.1016/j.enpol.2020.111578, Rev.energy policy,143,111578,( 2020). 